\title{
Construction Smooth Hausdroff Space from Fuzzy Metric Space
}

\author{
${ }^{1}$ Munir Abdul khalik Alkhafaji, ${ }^{2}$ Amer Himza Almyaly \\ ${ }^{1,2}$ the College of Education Department of Mathematics / Mustinsiryah University-Iraq
}

\begin{abstract}
In this paper, concepts of smooth topology and fuzzy metric space have been introduced. We define smooth Hausdroff space and so fuzzy open ball in fuzzy metric space to get the smooth topological space which is called induced smooth topological space then we proved this fuzzy metric is smooth Hausdroof space.
\end{abstract}

Keywords: Smooth topology, Fuzzy metric space, quasi $(q)$ and not quasi $(-q)$-coincident.

\section{Introduction:}

Many authors have introduced the concept of smooth topology on crisp set $[1,2,4]$ and so fuzzy metric space $[5,7]$.There are several definitions of fuzzy Metric space, one is using fuzzy numbers to define metric in ordinary spaces [6] and the other using fuzzy scalars (fuzzy points defined on the real-valued space R) to measure the distances between fuzzy points [5] as in this paper. In this paper, fuzzy points are usually denoted by $x_{\lambda}$ and the set of all the fuzzy points defined on $\mathrm{X}$ is denoted by $P_{F}(X)$. Particularly, when $\mathrm{X}=\mathrm{R}$, fuzzy points are also called fuzzy scalars and the set of all the fuzzy scalars is denoted by $S_{F}(R)$.

\section{1-Preliminaries}

Definition 1.1: [1, 2, And 4]: A smooth topological space (sts) is a pair $(\mathrm{X}, \tau)$ where $\mathrm{X}$ is a nonempty set and $\tau: I^{x} \rightarrow I$ is mapping satisfy the following properties:

$1-\tau(X)=\tau(\varnothing)=1$.

2- $\forall A, B \in I^{x}, \tau(A \cap B) \geq \tau(A) \wedge \tau(B)$.

3- For every subfamily $\left\{A_{i}: i \in J\right\} \subseteq I^{x}, \tau\left(\cup_{i \in J} A_{i}\right) \geq \Lambda_{i \in J} \tau\left(A_{i}\right)$.

Definition 1.2: let $X$ be a nonempty set and $\tau$ is st defines on it, the family $\tau_{\alpha}=\left\{A \in I^{x}: \tau(A) \geq \alpha\right\}$ and $\alpha \in$ $(0,1]$, is called $\alpha$-level.

Note: In the following when we say the set $\mathrm{G}$ is open or neighborhood then we meaning $\tau(G) \geq \alpha$ for some $\alpha \in$ $(0,1]$.

Definition 1.3: Let $(\mathrm{X}, \tau)$ be sts, $\left(X, \tau_{\alpha}\right)$ is fuzzy Hausdroff if for each distinct fuzzy points $x_{t}$ and $y_{r}$ in $\mathrm{X}$ when $x \neq y$ there exist fuzzy neighborhoods $G_{1}$ of $x_{t}$ and $G_{2}$ of $y_{r}$ such that $G_{1-} \mathrm{q} G_{2}$, when $x=y$ and $t<r$ (say) $x_{t}$ has a fuzzy neighborhoods $G_{1}$ and $y_{r}$ has a fuzzy q-neighborhoods $G_{2}$ in which $G_{1-\mathrm{q}} G_{2}$.

Definition 1.4: An sts $(X, \tau)$ is called "smooth Hausdroff" iff for each $\alpha \in(0,1],\left(X, \tau_{\alpha}\right)$ is fuzzy Hausdroff.

Definition 1.5, [7]: Suppose $a_{\lambda}$ and $b_{\gamma}$ are two fuzzy scalars then we say:

1- $a_{\lambda}$ No less than $b_{\gamma}$ if $a \geq b$ and denoted by $a_{\lambda}>b_{\gamma}$ or $b_{\gamma}<a_{\lambda}$.

2- $\quad a_{\lambda}$ Is nonnegative if $a \geq 0$. The set of all the nonnegative fuzzy scalars is denoted by $S_{F}^{+}(R)$.

Definition 1.6, [5]: Suppose $\mathrm{X}$ is a nonempty set and $d_{F}: P_{F} \times P_{F} \rightarrow S_{F}^{+}(R)$ is a mapping. ( $\left.P_{F}(X), d_{F}\right)$ Is said to be a fuzzy metric space if for any fuzzy points $x_{t}, y_{r}$ and $z_{p}$ belong to $P_{F}(X), d_{F}$ satisfies the following three conditions:

1- Nonnegative: $d_{F}\left(x_{t}, y_{r}\right)=0$ iff $\mathrm{x}=\mathrm{y}$ and $\mathrm{t}=\mathrm{r}=1$.

2- Symmetric: $d_{F}\left(x_{t}, y_{r}\right)=d_{F}\left(y_{r}, x_{t}\right)$.

3- Triangle inequality: $d_{F}\left(x_{t}, z_{p}\right) \prec\left(x_{t}, y_{r}\right)+\left(y_{r}, z_{p}\right)$.

\section{Smooth Metric Space.}

Definition 2.1: The fuzzy open ball $B_{\varepsilon_{\lambda}}\left(x_{t}\right)$ with fuzzy center $x_{t}$ and fuzzy radius $\varepsilon_{\lambda}, \varepsilon \in \mathbb{R}^{+}$defines as following: $B_{\varepsilon_{\lambda}}\left(x_{t}\right)=\left\{y_{r} \in X: r=t\right.$ and $\left.d_{F}\left(x_{t}, y_{r}\right) \prec \varepsilon_{\lambda}\right\}$.

Proposition 2.1: Let $\tau: I^{x} \rightarrow I$ is mapping define as following:

$$
\tau(B)=\left\{\begin{array}{c}
1 \text { if for any } x_{t} \in B \text { there exist fuzzy open ball } B_{\varepsilon_{\lambda}}\left(x_{t}\right) \subseteq B \\
0 \text { otherwise }
\end{array}\right.
$$


Then $\tau$ is st on fuzzy metric space $\left(P_{F}(X), d_{F}\right)$, since:

1- $\phi$ Has no elements therefore $\tau(\phi)=1$, and for any $x_{t} \in X$ there exist fuzzy open ball $B_{\varepsilon_{\lambda}}\left(x_{t}\right)$ belong to $\mathrm{X}$ therefore $\tau(X)=1$.

2- Let $A_{1}, A_{2} \in P(A)$ then:

a- If $A_{1}$ and $A_{2}$ are not open, if $A_{1}$ is open and $A_{2}$ is not open or conversely then it's clear $\tau\left(A_{1} \cap\right.$

$\left.A_{2}\right) \geq \tau\left(A_{2}\right) \wedge \tau\left(A_{2}\right)$.

b- If $A_{1}$ and $A_{2}$ are open then we want to prove $\tau\left(A_{1} \cap A_{2}\right) \geq \tau\left(A_{2}\right) \wedge \tau\left(A_{2}\right)$ :

Let $x_{t} \in A_{1} \cap A_{2} \Rightarrow x_{t} \in A_{1}$ and $x_{t} \in A_{2}$ then there exist two fuzzy open balls $B_{\varepsilon_{\lambda}}\left(x_{t}\right)$ and $B_{\delta_{\gamma}}\left(x_{t}\right)$ contain $x_{t}$, contained in $A_{1}$ and $A_{2}$, respectively, such that:

$B_{\varepsilon_{\lambda}}\left(x_{t}\right)=\left\{y_{r} \in X: r=t\right.$ and $\left.d_{F}\left(x_{t}, y_{r}\right) \prec \varepsilon_{\lambda}\right\}$

$B_{\delta_{\gamma}}\left(x_{t}\right)=\left\{z_{s} \in X: s=t\right.$ and $\left.d_{F}\left(x_{t}, z_{s}\right) \prec \delta_{\gamma}\right\}$

Let $l=\min \{r, s\}$ and $\Omega=\{\varepsilon, \delta\}$ then $B_{\Omega_{\Lambda}}\left(x_{t}\right)=\left\{h_{l} \in X: l=t\right.$ and $\left.d_{F}\left(x_{t}, h_{l}\right)<\Omega_{\Lambda}\right\} \Lambda=\lambda$ or $\gamma$. Since $l=\min \{r, s\}$ and $\Omega_{\Lambda}<\varepsilon_{\lambda}, \delta_{\gamma}$ then $B_{\Omega_{\Lambda}}\left(x_{t}\right) \subseteq B_{\varepsilon_{\lambda}}\left(x_{t}\right)$ and $B_{\Omega_{\Lambda}}\left(x_{t}\right) \subseteq B_{\delta_{\gamma}}\left(x_{t}\right) \Rightarrow B_{\Omega_{\Lambda}}\left(x_{t}\right) \subseteq$ $B_{\varepsilon_{\lambda}}\left(x_{t}\right) \cap B_{\delta_{\gamma}}\left(x_{t}\right) \Rightarrow \tau\left(A_{1} \cap A_{2}\right)=1$.

3- Let $\left\{A_{i}\right\}_{i \in I}$ be a collection of fuzzy subsets of A then if there exist $i \in I, A_{i}$ is not open $\Rightarrow \tau\left(\mathrm{U}_{i \in I} A_{i}\right) \geq$ $\bigwedge_{i \in I} \tau\left(A_{i}\right)$. Let for each $i \in I, A_{i}$ is open then we want to prove $\tau\left(\bigcup_{i \in I} A_{i}\right) \geq \bigwedge_{i \in I} \tau\left(A_{i}\right)$ :

Let $x_{t} \in \cup_{i \in I} A_{i} \Rightarrow \exists i \in I$ such that $x_{t} \in A_{i}$ but $A_{i}$ is open, then there exist fuzzy open ball $B_{\varepsilon_{\lambda}}\left(x_{t}\right) \subseteq$ $A_{i}$ but $A_{i} \subseteq \bigcup_{i \in I} A_{i} \Rightarrow B_{\varepsilon_{\lambda}}\left(x_{t}\right) \subseteq \bigcup_{i \in I} A_{i} \Rightarrow \tau\left(\bigcup_{i \in I} A_{i}\right) \geq \bigwedge_{i \in I} \tau\left(A_{i}\right)$.

Definition 2.2: Let $\left(P_{F}(X), d_{F}\right)$ be a fuzzy metric space and $\tau$ be a fuzzy st on $\left(P_{F}(X), d_{F}\right)$ then $\left(P_{F}(X), \tau\right)$ is called induced smooth topological space.

Proposition 2.2: The induced smooth topological space $\left(P_{F}(X), \tau\right)$ is smooth Hausdroff.

Proof: Let $x_{t}, y_{r} \in P_{F}(X)$, then:

1. If $x \neq y$, let $d_{F}\left(x_{t}, y_{r}\right)=\varepsilon_{\lambda}$ and we define $B_{\frac{\varepsilon}{2} \lambda}\left(x_{t}\right)=\left\{z_{s} \in X: s=t\right.$ and $\left.d_{F}\left(x_{t}, z_{s}\right)<\frac{\varepsilon}{2_{\lambda}}\right\}$ and so $B_{\frac{\varepsilon}{2}}\left(y_{r}\right)=$ $\left\{h_{l} \in X: l=r\right.$ and $\left.d_{F}\left(y_{r}, h_{l}\right)<\frac{\varepsilon}{2 \lambda}\right\}$.

Let $g_{k} \in B_{\frac{\varepsilon}{2} \lambda}\left(x_{t}\right) \cap B_{\frac{\varepsilon}{2} \lambda}\left(y_{r}\right) \Rightarrow k=t$ and $d_{F}\left(x_{t}, g_{k}\right)<\frac{\varepsilon}{2 \lambda}$ and so $k=r$ and $d_{F}\left(y_{r}, g_{k}\right)<\frac{\varepsilon}{2 \lambda} \Rightarrow d_{F}\left(x_{t}, y_{r}\right) \prec$ $\left(x_{t}, g_{k}\right)+\left(g_{k}, y_{r}\right)<\frac{\varepsilon}{2 \lambda}+\frac{\varepsilon}{2 \lambda}=\frac{\varepsilon}{22 \lambda}-\lambda^{2} \quad$ [3], but $\frac{\varepsilon}{22 \lambda}-\lambda^{2}<\varepsilon_{\lambda}$ and this contradiction, therefore $B_{\frac{\varepsilon}{2} \lambda}\left(x_{t}\right)-$ $q B_{\frac{\varepsilon}{2} \lambda}\left(y_{r}\right)$.

2. Let $\mathrm{x}=\mathrm{y}$ and $\mathrm{t}<r$.let $\mathrm{B}_{\varepsilon_{\lambda}}\left(\mathrm{x}_{\mathrm{t}}\right)=\left\{\mathrm{z}_{\mathrm{s}} \in \mathrm{X}: \mathrm{s}=\mathrm{t}\right.$ and $\left.\mathrm{d}_{\mathrm{F}}\left(\mathrm{x}_{\mathrm{t}}, \mathrm{z}_{\mathrm{s}}\right)<\varepsilon_{\lambda}\right\}$ and let $\mathrm{h}_{\mathrm{l}} \in \mathrm{X}$ s.t $\mathrm{l}+\mathrm{t} \leq 1$ and $\mathrm{l}+\mathrm{r}>1$ then we will define the following ball $B_{\varepsilon_{\lambda}}\left(h_{l}\right)=\left\{g_{k} \in X: k=l\right.$ and $\left.d_{F}\left(h_{l}, g_{k}\right)<\varepsilon_{\lambda}\right\}$ s.t $y_{r} \in B_{\varepsilon_{\lambda}}\left(h_{l}\right) \Rightarrow$ $\mathrm{B}_{\varepsilon_{\lambda}}\left(\mathrm{h}_{1}\right) \mathrm{qy}_{\mathrm{r}}$ and $\mathrm{B}_{\varepsilon_{\lambda}}\left(\mathrm{h}_{\mathrm{l}}\right)-\mathrm{qB}_{\varepsilon_{\lambda}}\left(\mathrm{x}_{\mathrm{t}}\right)$.

\section{Reference:}

[1]. C. Hua Yan, S. Zhang Guo, I-Fuzzy Topological Groups, Fuzzy Sets and Systems, V.161 (2010) 2166-2180.

[2]. H. Yia Li, F. Gui Shi, Some Separation Axioms in I-Fuzzy topological spaces, Fuzzy sets and Systems, V.159 (2008) $573-587$.

[3]. H. J. Zimmermann, Fuzzy set theory and its applications, K. Nijhoff, (1985), 29.

[4]. M. Demirci, Three Topological Structure of Smooth Topological Spaces, Fuzzy Sets and Systems, V.101 (1991), 185-190.

[5]. N. Kabir, Sh. Ghasemzadeh, Separable Fuzzy Metric Spaces, Mathematical Sciences, V.3, No.1 (2009) 55-62.

[6]. O. Kaleva and S. Seikkala, On fuzzy metric space, Fuzzy Sets and Systems 12 (1984), 215-229.

[7]. Z. Quan Xia and F. Fang GUO, Fuzzy Metric Spaces, J. Appl. Math. \& Computing, V.16, No. 1 - 2 (2004), 371 - 381. 